\title{
Termination of Cycle Rewriting
}

\author{
Hans Zantema ${ }^{1,2}$ and Barbara König ${ }^{3}$ and H. J. Sander Bruggink ${ }^{3}$ \\ 1 TU Eindhoven, Department of Computer Science, P.O. Box 513, \\ 5600 MB Eindhoven, The Netherlands h.zantema@tue.nl \\ 2 Radboud University Nijmegen, Institute for Computing and Information Sciences, \\ P.O. Box 9010, 6500 GL Nijmegen, The Netherlands \\ 3 Duisburg Essen University, Abteilung Informatik und Angewandte \\ Kognitionswissenschaft, 47048 Duisburg, Germany \\ barbara_koenig@uni-due.de sander.bruggink@uni-due.de
}

\begin{abstract}
String rewriting can not only be applied on strings, but also on cycles and even on general graphs. In this paper we investigate termination of string rewriting applied on cycles, shortly denoted as cycle rewriting, which is a strictly stronger requirement than termination on strings. Most techniques for proving termination of string rewriting fail for proving termination of cycle rewriting, but match bounds, arctic matrices and tropical matrices can be applied. Further we show how any terminating string rewriting system can be transformed to a terminating cycle rewriting system, preserving derivational complexity.
\end{abstract}

\section{Introduction}

A string rewriting systems (SRS) consists of a set of rules $\ell \rightarrow r$ where $\ell$ and $r$ are strings, that is, elements of $\Sigma^{*}$ for some alphabet $\Sigma$. String rewriting means that for such a rule $\ell \rightarrow r$ an occurrence of $\ell$ is replaced by $r$. In the standard interpretation this only works on strings: a string of the shape $u \ell v$ is replaced by urv. However, it is natural also to apply this on a cycle, that is, a string in which the start point is connected to its end point. For instance, for an SRS $R$ containing the rule $a b \rightarrow c b a$ we want to allow the cycle rewrite step
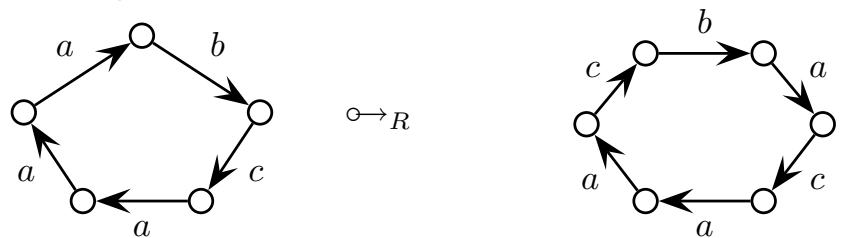

in which we use the notation $\hookrightarrow_{R}$ for a cycle rewrite step. In this paper we investigate termination of cycle rewriting. It is easy to see that termination of cycle rewriting implies termination of string rewriting. However, the other way around does not hold: the single rewrite rule $a b \rightarrow b a$ is terminating in the setting of string rewriting, but not in the setting of cycle rewriting, since the cycle $a b$ of length 2 rewrites to the cycle $b a$ which is equal to the cycle $a b$, so this rewriting can go on forever. 
Cycle rewriting can be seen as a special instance of graph transformation. In a separate paper [1] we investigate how the techniques of this paper extend to the general setting of graph transformation. In particular there we show that for a graph transformation system in which all rules are string rewrite rules, termination on all cycles coincides with termination on all graphs. So from the perspective of termination of graph transformation it is more natural to consider string rewriting to be applied on cycles rather than on strings, justifying an investigation of cycle rewriting as a separate topic. Moreover, cycles are often used in communication protocols with message passing on a ring structure, and in that setting steps are essentially cycle rewrite steps.

Standard techniques for proving termination of term rewriting and string rewriting like recursive path order, dependency pairs and polynomial interpretation fail to prove termination of cycle rewriting as they all easily prove termination of the single rule $a b \rightarrow b a$ which is not terminating in the cycle setting. They all exploit the term structure by which every string has a begin and an end, while a cycle has not. Nevertheless, for a few other powerful techniques, in particular match bounds, arctic matrices and tropical matrices, we show that these can be applied to prove termination of cycle rewriting. It turns out that the techniques of arctic and tropical matrices can be interpreted by weighting in type graphs: tropical matrices correspond to the requirement that every morphism of a left hand side to the type graph admits a morphism of the corresponding right hand side to the type graph of a lower weight, while arctic matrices correspond to the requirement that every morphism of a right hand side to the type graph admits a morphism of the corresponding left hand side to the type graph of a higher weight. Further match bound proofs can be seen as a particular case of a tropical matrix proof. We developed an implementation automatically finding proofs based on a combination of these techniques.

We investigate derivational complexity of cycle rewrite systems for which termination is proved by these techniques. Arctic matrices and match bounds have been exploited before for proving bounds on derivational complexity of term rewriting and string rewriting: single applications of these techniques yield linear bounds, while combined application may yield higher bounds. In this paper we give similar results in the setting of cycle rewriting. In particular we give examples of length preserving systems for which termination can be proved by combining the above mentioned techniques, while any polynomial can be achieved as a lower bound for derivational complexity. For non-length-preserving systems we show that exponential derivational complexity can be reached.

We investigate a particular shape of SRSs for which we show that termination of string rewriting and cycle rewriting coincide; it is characterized by end symbols that only occur as the last symbol of a left hand side or right side of a rule. We show how any SRS can be transformed to an SRS of this special shape, preserving termination and derivational complexity. As a consequence, termination of cycle rewriting is undecidable, and for every computable function an SRS $R$ exists for which cycle rewriting is terminating and the derivational complexity exceeds the computable function. 
The paper is organized as follows. Section 2 presents basic definitions and observations related to cycle rewriting. Section 3 presents the techniques for proving termination of cycle rewriting. In Section 4 derivational complexity of these techniques is investigated. Section 5 introduces the special format with end symbols and presents the corresponding theory. In Section 6 our implementation is discussed. We conclude in Section 7.

\section{Cycle Rewriting}

We consider cycles over an alphabet $\Sigma$ which are essentially strings over $\Sigma$ in which the leftmost element is connected to the rightmost element. We represent cycles by strings, where for all strings $u, v$ the string $u v$ represents the same cycle as $v u$. More precisely, for any alphabet $\Sigma$ we define the set Cycle $(\Sigma)$ of cycles over $\Sigma$ by

$$
\operatorname{Cycle}(\Sigma)=\Sigma^{*} / \sim
$$

where $\sim$ is the equivalence relation on $\Sigma^{*}$ defined by

$$
u \sim v \Longleftrightarrow \exists u_{1}, u_{2} \in \Sigma^{*}: u=u_{1} u_{2} \wedge v=u_{2} u_{1} .
$$

It is straightforward to check that indeed $\sim$ is an equivalence relation. The cycle represented by a string $u$, i.e., the equivalence class of $u$ w.r.t $\sim$, is denoted by $[u]$.

As usual we define a string rewrite system (SRS) over $\Sigma$ to be a subset $R$ of $\Sigma^{*} \times \Sigma^{*}$. Elements $(\ell, r)$ of an SRS are called (string rewrite) rules and are usually written as $\ell \rightarrow r$, where $\ell$ is called the left hand side (lhs) and $r$ the right hand side (rhs) of the rule. As usual, the string rewrite relation $\rightarrow_{R}$ on $\Sigma^{*}$ is defined by $u \rightarrow_{R} v \Longleftrightarrow \exists x, y \in \Sigma^{*}, \ell \rightarrow r \in R: u=x \ell y \wedge v=x r y$.

For an SRS $R$ over $\Sigma$ we define the corresponding cycle rewrite relation $\hookrightarrow \rightarrow R$ on $\operatorname{Cycle}(\Sigma)$ as follows:

$$
[u] \circ \rightarrow_{R}[v] \Longleftrightarrow \exists x \in \Sigma^{*}, \ell \rightarrow r \in R: \ell x \sim u \wedge r x \sim v .
$$

Equivalently, one can state $[u] a_{R}[v] \Longleftrightarrow \exists u^{\prime} \in[u], v^{\prime} \in[v]: u^{\prime} \rightarrow_{R} v^{\prime}$.

The main goal of this paper is to study $a_{R}$, in particular how to prove termination.

Lemma 1 Let $R$ be an SRS over an alphabet $\Sigma$ for which the relation $\hookrightarrow_{R}$ on Cycle $(\Sigma)$ is terminating. Then the string rewrite relation $\rightarrow_{R}$ on $\Sigma^{*}$ is terminating too.

Proof. Assume $u \rightarrow_{R} v$ for $u, v \in \Sigma^{*}$. Then $u=x \ell y$ and $v=x r y$ for some $x, y \in \Sigma^{*}, \ell \rightarrow r \in R$. From $u \sim \ell y x$ and $v \sim r y x$ we conclude $[u]_{\circ} \rightarrow_{R}[v]$. Hence an infinite $\rightarrow R$-reduction transforms to an infinite $\leftrightarrow \rightarrow_{R}$-reduction, proving the lemma. 
The converse of Lemma 1 does not hold: the SRS consisting of the single rule $a b \rightarrow b a$ is clearly terminating, but since $[a b]=[b a]$ the corresponding cycle rewrite relation $a$ is not terminating.

So termination of $\hookrightarrow_{R}$ is a stronger requirement than termination of $\rightarrow_{R}$.

A next natural question to ask is how confluence of $\hookrightarrow_{R}$ is related to confluence of $\rightarrow_{R}$. It turns out that none of the possible implications holds. As a first example consider the SRS consisting of the two rules $a b \rightarrow b a, a b \rightarrow b$. Since the string $a b$ rewrites to both $b a$ and $b$, both being normal forms, the relation $\rightarrow_{R}$ is not confluent for this SRS. However, with respect to $\hookrightarrow_{R}$ every string containing $n b$-s rewrites to $b^{n}$ while every string containing no $b$-s is a normal form, hence $a_{R}$ is confluent. Conversely consider the SRS consisting of the two rules $a b \rightarrow a a, \quad b a \rightarrow b b$. Straightforward critical pair analysis shows that $\rightarrow_{R}$ is locally confluent; since it is terminating (proved e.g. by dependency pairs) it is confluent too. However, $a_{R}$ is neither confluent since $a b$ admits two normal forms $a a$ and $b b$, nor terminating since $a a b \sim a b a \rightarrow_{R} a b b \rightarrow_{R} a a b$.

Also with respect to weak normalization the relations $a_{R}$ and $\rightarrow_{R}$ are incomparable: for the single rule $a b \rightarrow b a$ the relation $\rightarrow_{R}$ is weakly normalizing, while $a_{R}$ is not. Conversely, for the SRS consisting of the two rules $a b \rightarrow$ $a b, b a \rightarrow a$ the relation $a_{R}$ is weakly normalizing, while $\rightarrow_{R}$ is not.

\section{Termination by Type Graphs}

From now on we concentrate on developing techniques to prove termination of $a_{R}$. We start by a straightforward approach to consider decreasing weights. A weight function $W: \Sigma \rightarrow \mathbb{N}$ is extended to a weight function $W: \Sigma^{*} \rightarrow \mathbb{N}$ by defining inductively $W(\epsilon)=0$ and $W(a x)=W(a)+W(x)$ for $a \in \Sigma, x \in \Sigma^{*}$ : the weight of a string is simply the sum of the weights of its elements.

Lemma 2 Let $R$ be an SRS over $\Sigma$ and let $W: \Sigma \rightarrow \mathbb{N}$ satisfy

- W( $\ell) \geq W(r)$ for all $\ell \rightarrow r \in R$, and

- $\rightarrow R^{\prime}$ is terminating for $R^{\prime}=\{\ell \rightarrow r \in R \mid W(\ell)=W(r)\}$.

Then $\hookrightarrow_{R}$ is terminating.

Proof. We prove that $W(u)=W(v)$ for all $u, v$ satisfying $[u] \circ \rightarrow R^{\prime}[v]$ and $W(u)>W(v)$ for all $u, v$ satisfying $[u] \circ \rightarrow_{R \backslash R^{\prime}}[v]$. Then termination of $R$ follows from termination of $R^{\prime}$ and well-foundedness of $>$. So let $[u] \hookrightarrow \hookrightarrow R \backslash R^{\prime}[v]$, then we can write $u=u_{1} u_{2}, v=v_{1} v_{2}, u_{2} u_{1}=\ell x, v_{2} v_{1}=r x$ for some $\ell \rightarrow r \in R \backslash R^{\prime}$. Then

$$
\begin{aligned}
W(u) & =W\left(u_{1} u_{2}\right)=W\left(u_{1}\right)+W\left(u_{2}\right)=W\left(u_{2} u_{1}\right) \\
& =W(\ell x)=W(\ell)+W(x) \\
& >W(r)+W(x)=W(r x) \\
& =W\left(v_{2} v_{1}\right)=W\left(v_{2}\right)+W\left(v_{1}\right)=W\left(v_{1} v_{2}\right)=W(v) .
\end{aligned}
$$

For the remaining case $[u] \circ \rightarrow R^{\prime}[v]$ we obtain exactly the same derivation with '>' replaced by '=', hence concluding $W(u)=W(v)$ which we had to prove. 
In simple applications of Lemma 2 we have $W(\ell)>W(r)$ for all $\ell \rightarrow r \in R$, by which $R^{\prime}$ is empty and hence $\hookrightarrow_{R}$ is trivially terminating. Then the only thing to be done for proving termination of $a_{R}$ is choosing a $W(a) \in \mathbb{N}$ for every $a \in \Sigma$ such that $W(\ell)>W(r)$ for all $\ell \rightarrow r \in R$.

Example 3 For the SRS $R$ consisting of the four rules

$$
a a \rightarrow b c, b b \rightarrow c d, c c \rightarrow d d d, d d d \rightarrow a c
$$

the relation $a_{R}$ is terminating due to Lemma 2 by choosing $W(a)=30, W(b)=$ $27, W(c)=32$ and $W(d)=21$, for which it is checked that $W(\ell)>W(r)$ for all four rules $\ell \rightarrow r$. These numbers are the smallest possible.

Next we give a generalization of Lemma 2 inspired by the notion of type graph as it appears in graph transformation systems [2] and coinciding with the approach of tropical and arctic matrix interpretations.

We define a type graph $(V, E, W)$ over a signature $\Sigma$ to be a directed graph in which the edges are labeled by symbols from $\Sigma$, and have a weight $W(e) \in \mathbb{N}$, that is, $E \subseteq V \times \Sigma \times V$ and $W: E \rightarrow \mathbb{N}$.

For $u=a_{1} a_{2} \cdots a_{n} \in \Sigma^{+}$and $p, q \in V$ we define a u-path from $p$ to $q$ in a type graph $(V, E, W)$ to be a sequence $\left(p_{1}, a_{1}, q_{1}\right)\left(p_{2}, a_{2}, q_{2}\right) \cdots\left(p_{n}, a_{n}, q_{n}\right)$ of edges in $E$ such that $p_{1}=p, q_{n}=q$ and $q_{i}=p_{i+1}$ for $i=1, \ldots, n-1$. The weight $W(u)$ of such a $u$-path is defined to be $\sum_{i=1}^{n} W\left(p_{i}, a_{i}, q_{i}\right)$. In case $p=q$, the $u$-path is called a $u$-cycle.

We distinguish two kinds of criteria to conclude $\hookrightarrow_{R}$ termination from morphisms from paths to type graphs: tropical and arctic. Tropical means that every path morphism of a left hand side to the type graph admits a morphism of the corresponding right hand side to the type graph of a lower weight, while arctic means that every path morphism of a right hand side to the type graph admits a morphism of the corresponding left hand side to the type graph of a higher weight. This terminology is inspired by the corresponding terminology for matrix interpretations.

Theorem 4 Let $R^{\prime} \subseteq R$ be SRSs over $\Sigma$. Let $(V, E, W)$ be a type graph over इ. Assume

- there exists $p \in V$ such that $(p, a, p) \in E$ for all $a \in \Sigma$, and

$-\circ R_{R^{\prime}}$ is terminating, and

- either

- (tropical) for every $\ell \rightarrow r \in R, p, q \in V$ and for every $\ell$-path from $p$ to $q$ in $(V, E, W)$ having weight $w$ there is an r-path from $p$ to $q$ in $(V, E, W)$ having weight $w^{\prime}$ with $w \geq w^{\prime}$, and $w>w^{\prime}$ if $\ell \rightarrow r \notin R^{\prime}$, or

- (arctic) for every $\ell \rightarrow r \in R, p, q \in V$ and for every $r$-path from $p$ to $q$ in $(V, E, W)$ having weight $w$ there is an $\ell$-path from $p$ to $q$ in $(V, E, W)$ having weight $w^{\prime}$ with $w^{\prime} \geq w$, and $w^{\prime}>w$ if $\ell \rightarrow r \notin R^{\prime}$.

Then $\hookrightarrow_{R}$ is terminating, and any $\hookrightarrow_{R}$ reduction of a cycle [u] contains at most $|u| \cdot w$ steps with respect to $R \backslash R^{\prime}$ for $w=\max _{e \in E} W(e)$. 
Proof. Assume $\left[u_{0}\right] \hookrightarrow_{R}\left[u_{1}\right] \hookrightarrow_{R}\left[u_{2}\right] \hookrightarrow_{R} \cdots \hookrightarrow_{R}\left[u_{n}\right]$ contains more than $\left|u_{0}\right| \cdot$ $w$ steps with respect to $R \backslash R^{\prime}$ for $w=\max _{e \in E} W(e)$. We will derive a contradiction; this proves the theorem as termination immediately follows.

For the tropical case choose any $u_{0}$-cycle; this exists due to the first assumption of the theorem. Let $w_{0}$ be the weight of this cycle.

Next, for $i=1,2,3, \ldots$ we can choose a $u_{i}$-cycle by replacing the $\ell$-path being the part of the $u_{i-1}$-cycle by the corresponding $r$-path as indicated in the tropical condition of the theorem, where $\ell \rightarrow r$ is the rule applied in $\left[u_{i-1}\right] a_{R}\left[u_{i}\right]$. Let $w_{i}$ be the weight of this new cycle; if $\ell \in r \in R \backslash R^{\prime}$ then $w_{i}<w_{i-1}$, otherwise $w_{i} \leq w_{i-1}$. As by the assumption there are more than $\left|u_{0}\right| \cdot w$ steps with ' $<$ ', and $w_{n} \geq 0$, we conclude $w_{0}>\left|u_{0}\right| \cdot w$. This contradicts the definition of $w=\max _{e \in E} W(e)$.

For the arctic case choose any $u_{n}$-cycle; this exists due to the first assumption of the theorem. Let $w_{n}$ be the weight of this cycle.

Next, for $i=n-1, n-2, n-3, \ldots$ we can choose a $u_{i}$-cycle by replacing the $r$-path being the part of the $u_{i+1}$-cycle by the corresponding $r$-path as indicated in the arctic condition of the theorem, where $\ell \rightarrow r$ is the rule applied in $\left[u_{i}\right] \rightarrow_{R}\left[u_{i+1}\right]$. Let $w_{i}$ be the weight of this new cycle; if $\ell \in r \in R \backslash R^{\prime}$ then $w_{i}<w_{i-1}$, otherwise $w_{i} \leq w_{i-1}$. The rest of the argument is as before.

In case the type graph consist of a single node, every path in this type graph consists of a sequence of edges of this node to itself. In this case the conditions for the tropical case and the termination conclusion of Theorem 4 coincide with the conditions and the conclusion of Lemma 2. Hence indeed we can state that Theorem 4 is a generalization of Lemma 2. The next example shows that it is a strict generalization.

Example 5 For the SRS $R$ consisting of the single rule $a a \rightarrow a b a$ Lemma 2 does not apply, since $2 W(a)>2 W(a)+W(b)$ has no solutions in the natural numbers. Instead we define a type graph consisting of two nodes 1 and 2 , and four edges $(1, a, 1),(1, b, 1),(1, a, 2),(2, b, 1), \quad$ of which $(1, a, 1)$ has weight 1 and all others have weight 0 , as indicated in the picture.

Now there are exactly two $a a$-paths:

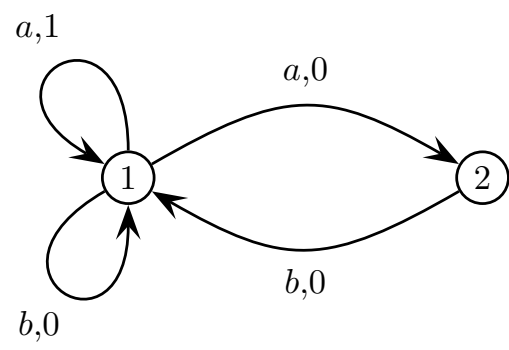

$-1 \rightarrow_{a} 1 \rightarrow_{a} 1$ of weight 2 that may be replaced by the $a b a$-path $1 \rightarrow_{a} 2 \rightarrow_{b}$ $1 \rightarrow_{a} 1$ of weight 1 , and

$-1 \rightarrow_{a} 1 \rightarrow_{a} 2$ of weight 1 that may be replaced by the $a b a$-path $1 \rightarrow_{a} 2 \rightarrow_{b}$ $1 \rightarrow_{a} 2$ of weight 0 .

So all conditions of the tropical version of Theorem 4 are satisfied in choosing $R^{\prime}$ to be empty, proving that $\hookrightarrow_{R}$ is terminating.

Type graphs can be represented by matrices in the following natural way. Number the nodes of the type graphs from 1 to $n$. For every $a \in \Sigma$ let $A_{a}$ be 
the matrix such that $A_{a}(i, j)=w$ if and only if an edge $(i, a, j)$ exists of weight $w$, while $A_{a}(i, j)=\infty$ if no such edge exist. So for example, the type graph of Example 5 is represented by the following matrices:

$$
A_{a}=\left(\begin{array}{cc}
1 & 0 \\
\infty & \infty
\end{array}\right), A_{b}=\left(\begin{array}{cc}
0 & \infty \\
0 & \infty
\end{array}\right)
$$

Now we consider the semiring $(\mathbb{N} \cup\{\infty\}$, min, + ), that is, the semiring consisting of $\mathbb{N} \cup\{\infty\}$ on which the binary operator min acts as the semiring addition and the normal addition acts as the semiring multiplications. Here on $\mathbb{N}$ the operators min and + act as usual, while it is extended to $\mathbb{N} \cup\{\infty\}$ by defining

$$
\min (\infty, x)=\min x, \infty=x \text { and } \infty+x=x+\infty=\infty
$$

for all $x \in \mathbb{N} \cup\{\infty\}$. Now $\infty$ acts as the semiring zero and 0 acts as the semiring unit. This semiring is called the tropical semiring after its study by the Brazilian mathematician Imre Simon [7]. Now it is easily checked that path concatenation corresponds to matrix multiplication over this semiring, more precisely, if $A_{u}$ is defined by $A_{u}(i, j)$ to be the lowest weight of a $u$-path from $i$ to $j$, and $\infty$ if no such path exists, then $A_{u v}=A_{u} \times A_{v}$, where $\times$ is matrix multiplication with respect to this semiring. For instance, in the above example we have $A_{a b}=$ $\left(\begin{array}{cc}0 & \infty \\ \infty & \infty\end{array}\right)$. In this notation the tropical condition can be reformulated to $A_{\ell} \geq A_{r}$ for all $\ell \rightarrow r \in R$ and $A_{\ell}>A_{r}$ for all $\ell \rightarrow r$ not in $R^{\prime}$. Here $\geq$ and $>$ on matrices are defined by

$$
A \geq B \Longleftrightarrow \forall i, j: A(i, j) \geq B(i, j), \quad A>B \Longleftrightarrow \forall i, j: A(i, j)>B(i, j),
$$

in which $\geq$ and $>$ on $\mathbb{N}$ is extended to $\mathbb{N} \cup\{\infty\}$ by defining $\infty \geq x$ and $\infty>x$ for all $x \in \mathbb{N} \cup\{\infty\}$. Note that this also yields $\infty>\infty$, by which $>$ is not well-founded on the full set $\mathbb{N} \cup\{\infty\}$.

Indeed, in example 5 we have

$$
A_{a a}=\left(\begin{array}{cc}
2 & 1 \\
\infty & \infty
\end{array}\right)>A_{a b a}=\left(\begin{array}{cc}
1 & 0 \\
\infty & \infty
\end{array}\right)
$$

Also the arctic condition can be described by a matrix condition over a semiring: the arctic semiring $(\mathbb{N} \cup\{-\infty\}, \max ,+)$, so similar to the tropical semiring, but now with max as the semiring addition, having $-\infty$ as its zero, in which $-\infty$ is less than all other elements. In this notation the arctic condition can be reformulated to $A_{\ell} \geq A_{r}$ for all $\ell \rightarrow r \in R$ and $A_{\ell}>A_{r}$ for all $\ell \rightarrow r$ not in $R^{\prime}$. These arctic matrix interpretations have been studied in [6], being a modification of matrix interpretations [3]. There the termination proofs for term rewriting (including string rewriting) are based on monotone algebras. For our cycle setting the monotone algebra approach failed, but by the type graph approach we succeeded to give termination proofs for both the tropical and arctic condition. 
This section is concluded by showing how the method of match-bounds for proving termination of string rewriting can be seen as a special instance of tropical type graphs, and therefore also proves termination of cycle rewriting. Here we refer to the basic version of match-bounds which is also used for proving linear derivational complexity of string rewriting, and not the version based on forward closures which is more powerful for proving termination of string rewriting. Surprisingly, this basis theorem of match-bounds uses exactly the same data structure of a type graph: a directed graph in which every edge is labeled by a symbols from $\Sigma$ and has a natural number assigned to it. Where in type graphs this natural number serves as a weight, denoted by $W$, in match-bounds it serves as a height and is denoted by $H$.

Theorem 6 Let $R$ be SRSs over $\Sigma$. Let $(V, E, H)$ be a type graph over $\Sigma$. Assume

- there exists $p \in V$ such that $(p, a, p) \in E$ for all $a \in \Sigma$ and $H(p, a, p)=0$, and

- for every $\ell \rightarrow r \in R, p, q \in V$ and for every $\ell$-path from $p$ to $q$ in $(V, E, H)$ there is an $r$-path from $p$ to $q$ in $(V, E, H)$ such that the height of every edge in this r-path is $1+m$, where $m$ is the smallest height of an edge in the $\ell$-path.

Then $\hookrightarrow_{R}$ is terminating.

If the conclusion of Theorem 6 is weakened to termination of $\rightarrow_{R}$, it coincides to the basic version of the match-bound theorem for string rewriting from $[4,9]$.

Proof. For proving Theorem 6 we apply the tropical case of Theorem 4, in which we define $W(u, a, v)=s^{h-H(u, a, v)}$, where $s$ is a number higher than the length of the longest right hand side of $R$, and $h$ is the highest value of $H$ occurring in the type graph $(V, E, H)$. We choose $R^{\prime}=\emptyset$. Then all conditions of Theorem 4 hold, where the tropical condition for a rule $\ell \rightarrow r$ and an $\ell$-path from $u$ to $v$ with smallest height $m$ follows from

$$
W(\ell) \geq s^{h-m}>|r| \cdot s^{h-(m+1)}=W(r),
$$

in which the $r$ path from $u$ to $v$ is chosen according to the second condition of Theorem 6. So $\hookrightarrow_{R}$ is terminating according to Theorem 4 .

The typical use of match-bounds is that one tries to construct a corresponding type graph by completion: start by a single node with an $a$-loop of height 0 for every $a \in \Sigma$, and complete it by continue investigating all $\ell$-paths in the graph and add a corresponding $r$-path if it does not yet exist. If this process ends, termination has been proved. Note that both conditions of Theorem 6 can be weakened while keeping the same proof: in the first condition the requirement $H(p, a, p)=0$ may be removed since it is not used, and in the second condition an $r$-path with total weight less than the weight of the $\ell$-path is sufficient, also if not all edges in the $r$-path have height exactly $m+1$. 


\section{Derivational Complexity}

In term rewriting and string rewriting derivational complexity of a terminating rewrite system is defined to be the longest reduction length expressed in the size of the initial term. For cycle rewriting we do exactly the same: for an SRS $R$ over $\Sigma$ we define

$$
\mathrm{dc}_{R}(n)=\max \left\{k\left|\exists t, s \in \Sigma^{*}:\right| t \mid \leq n \wedge t \hookrightarrow_{R}{ }^{k} s\right\} .
$$

An SRS $R$ is said to have linear (quadratic, cubic, ...) derivational complexity with respect to cycle rewriting if $\mathrm{dc}_{R}(n)=\Theta(n)\left(\Theta\left(n^{2}\right), \Theta\left(n^{3}\right), \ldots\right)$.

Our first theorem on derivational complexity states that combined application of Lemma 2 only proves termination of systems with linear derivational complexity.

Theorem 7 Let $n \geq 1$. Let $R=\bigcup_{i=1}^{n} R_{i}$ for which for every $k=1, \ldots, n$ termination of $\bigcup_{i=1}^{k} R_{i}$ is proved by Lemma 2 by choosing $R^{\prime}=\emptyset$ for $k=1$ and $R^{\prime}=\bigcup_{i=1}^{k-1} R_{i}$ for $k>1$. Then $\mathrm{dc}_{R}(n)=O(n)$.

Proof. We apply induction on $n$. If $n=1$ then Lemma 2 is applied with $R^{\prime}=\emptyset$, meaning that $W(\ell)>W(r)$ for all $\ell \rightarrow r \in R$, from which we obtain $W(t)>$ $W\left(t^{\prime}\right)$ for every $t \mathrm{a}_{R} t^{\prime}$. If $C$ is the highest value of $W(a)$ for $a \in \Sigma$. Then from $t \circ \rightarrow R{ }^{k} s$ we conclude $k \leq W(t) \leq C|t|$, proving $\mathrm{dc}_{R}(n)=O(n)$.

For $n>1$ the termination proof of $R_{1} \cup R_{2}$ is given by weights $W_{1}$ and $W_{2}$ satisfying $W_{1}(\ell)>W_{1}(r)$ for $\ell \rightarrow r \in R_{1}, W_{2}(\ell)=W_{2}(r)$ for $\ell \rightarrow r \in R_{1}$, and $W_{2}(\ell)>W_{2}(r)$ for $\ell \rightarrow r \in R_{2}$. choose $C \in \mathbb{N}$ such that $C>W_{1}(\ell)-W_{1}(r)$ for all $\ell \rightarrow r \in R_{2}$. Define $W(a)=C W_{2}(a)+W_{1}(a)$ for $a \in \Sigma$, then combining the above properties yields $W(\ell)>W(r)$ for all $\ell \rightarrow r \in R_{1} \cup R_{2}$. So termination of $R_{1} \cup R_{2}$ is proved by Lemma 2 by choosing $R^{\prime}=\emptyset$ and the new weight $W$. Now the theorem follows by applying the induction hypothesis on $R=\bigcup_{i=1}^{n-1} R_{i}^{\prime}$ in which $R_{1}^{\prime}=R_{1} \cup R_{2}$ and $R_{k}^{\prime}=R_{k+1}$ for $k=2, \ldots, n-1$.

If termination of $a_{R}$ is proved by applying Theorem 4 for $R^{\prime}=\emptyset$, then $\mathrm{dc}_{R}(n)=O(n)$ as immediately follows from Theorem 4. In particular, this holds for proofs by match-bounds via Theorem 6 , as Theorem 6 was proved by applying Theorem 4 for $R^{\prime}=\emptyset$. However, by combined application of Theorem 4 much longer derivation lengths can be achieved: even by combining a single application of Theorem 4 with a single application of Lemma 2 exponential derivation lengths can be obtained, as is shown in the following example.

Example 8 Let the SRS $R$ consist of the four rules

$$
a L \rightarrow L b b, R b \rightarrow a R, B L \rightarrow R, R B \rightarrow L B .
$$

One easily shows that $B a^{k} L B$ rewrites to $a^{2 k} L B$ for every $k \geq 0$, so $B^{k} a L B$ rewrites to $a^{2^{k}} L B$. As the increase of size is exponential in the size of the original string, it has at least exponential derivation length: $\mathrm{dc}_{R}(n)=\Omega\left(2^{n}\right)$. Note that 
the reduction does not exploit the cycle structure, so the exponential derivation length both holds for cycle rewriting and string rewriting.

For proving termination of $a_{R}$ we apply Lemma 2 by choosing $W(B)=2$, $W(R)=1$ and $W(a)=W(b)=W(L)=0$. As $W(\ell)>W(r)$ for the last two rules $\ell \rightarrow r$, and $W(\ell)=$ $W(r)$ for the first two rules, it remains to prove termination for the first two rules. For doing so we apply the tropical case of Theorem 4 for $R^{\prime}=\emptyset$ and the following type graph: It contains the following paths labeled by left hand sides:

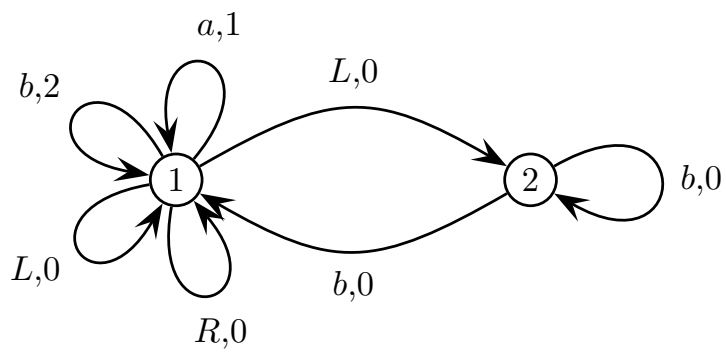

$-1 \rightarrow_{a} 1 \rightarrow_{L} 1$ of weight 1 to be replaced by $1 \rightarrow_{L} 2 \rightarrow_{b} 2 \rightarrow_{b} 1$ of weight 0 , $-1 \rightarrow_{a} 1 \rightarrow_{L} 2$ of weight 1 to be replaced by $1 \rightarrow_{L} 2 \rightarrow_{b} 2 \rightarrow_{b} 2$ of weight 0 , $-1 \rightarrow_{R} 1 \rightarrow_{b} 1$ of weight 2 to be replaced by $1 \rightarrow_{a} 1 \rightarrow_{R} 1$ of weight 1 ,

by which all requirements of Theorem 4 hold and termination of $\hookrightarrow_{R}$ can be concluded.

In a first view the following observations look quite contradictory to the observation that $R$ has exponential derivation length. The first two rules have linear derivation lengths since we found a proof by Theorem 4 in which $R^{\prime}=\emptyset$, and by the application of Lemma 2 we concluded that the number of applications of the other two rules is linear in the size of the original string. But the example clearly shows what is going on: between consecutive applications of the third and fourth rule the length of the string is doubled, and after doubling a linear of number times the length has increased to exponential size, after which the first two rules can be applied an exponential number of times.

The following theorem states that the situation is quite different if the lengths of the strings do not increase. An SRS $R$ is called length-non-increasing if $|r| \leq|\ell|$ for all $\ell \rightarrow \in R$.

Theorem 9 Let $R$ be a length-non-increasing $S R S$ and let $R^{\prime} \subseteq R$ satisfy all requirements of Theorem 4, and assume $\mathrm{dc}_{R^{\prime}}(n)=O(f(n))$ for some function $f$. Then $\mathrm{dc}_{R}(n)=O(n f(n))$.

Proof. Take an arbitrary $\hookrightarrow_{R}$ reduction $\left[u_{0}\right] \hookrightarrow_{R}\left[u_{1}\right] \hookrightarrow_{R} \cdots \hookrightarrow_{R}\left[u_{k}\right]$ of length $k$ starting with a string $u_{0}$ for which $\left|u_{0}\right|=n$. Since $R$ is length-non-increasing we obtain $\left|u_{i}\right| \leq n$ for all $i=0, \ldots, k$. From the proof of Theorem 4 we conclude that the total number of $\leftrightarrow \rightarrow R \backslash R^{\prime}$-steps in this reduction is at most $C n$ for some constant $C$. From $\left|u_{i}\right| \leq n$ for all $i=0, \ldots, k$ and $\mathrm{dc}_{R^{\prime}}(n)=O(f(n))$ we conclude that the maximal number of consecutive $\leftrightarrow R^{\prime}$-steps in this reduction is at most $D f(n)$ for some constant $D$. Combining these observations yields $k \leq C n+(1+C n) D f(n)$, from which we conclude $k=O(n f(n))$. 
So as a consequence, we conclude that for a length-non-increasing SRS $R$ termination of $a_{R}$ is proved only by consecutive application of Theorem 4, then $\mathrm{dc}_{R}(n)=O\left(n^{k}\right)$ for $k$ being the number of consecutive applications: $R$ has polynomial derivational complexity. Next we give an example showing that every polynomial derivational complexity can be achieved by length-non-increasing systems for which termination can be proved by repeated application of Theorem 4 .

Example 10 For $k \geq 1$ let $R_{k}$ be the union of

$$
f_{i} a_{0} \rightarrow a_{i} f_{i}, f_{i} \rightarrow f_{i-1}, a_{i} f_{0} \rightarrow f_{i-1} a_{0},
$$

for $i$ running from 1 to $k$. Let $F(n, i)$ be the number of steps of a particular reduction from $f_{i} a_{0}^{n}$ to $f_{0} a_{0}^{n}$. We will prove $F(n, k)=\Theta\left(n^{k}\right)$, from which $\mathrm{dc}_{R_{k}}=$ $\Theta\left(n^{k}\right)$ immediately follows, both in the setting of string rewriting and cycle rewriting. Due to $f_{1} a_{0}^{n} \rightarrow^{n} a_{1}^{n} f_{1} \rightarrow a_{1}^{n} f_{0} \rightarrow^{n} f_{0} a_{0}^{n}$ we obtain $F(n, 1)=2 n+1$. For $i>1$ we consider the reduction

$$
\begin{aligned}
f_{i} a_{0}^{n} \rightarrow^{n} a_{i}^{n} f_{i} \rightarrow^{i} a_{i}^{n} f_{0} & \rightarrow a_{i}^{n-1} f_{i-1} a_{0} \rightarrow^{F(1, i-1)} a_{i}^{n-1} f_{0} a_{0} \\
& \rightarrow a_{i}^{n-2} f_{i-1} a_{0}^{2} \rightarrow^{F(2, i-1)} a_{i}^{n-2} f_{0} a_{0}^{2} \\
& \rightarrow a_{i}^{n-3} f_{i-1} a_{0}^{3} \rightarrow^{F(3, i-1)} a_{i}^{n-3} f_{0} a_{0}^{3} \\
& \cdots \\
& \rightarrow f_{i-1} a_{0}^{n} \rightarrow^{F(n, i-1)} f_{0} a_{0}^{n},
\end{aligned}
$$

yielding $F(n, i) \geq \sum_{j=1}^{n} F(j, i-1)$. Now one proves $F(n, i)>(1 / i !) n^{i}$ by induction on $i$, using the well-known property $\sum_{j=1}^{n} j^{i-1} \geq(1 / i) * n^{i}$, concluding the proof.

Next we prove termination of $a R_{k}$ by repeated application of Theorem 4 and its special instance Lemma 2 . First remove $f_{k} \rightarrow f_{k-1}$ by counting $f_{k}$, that is, choose $W\left(f_{k}\right)=1$ and $W(a)=0$ for all $a \neq f_{k}$ in Lemma 2. Next apply

Theorem 4 by choosing the following type graph, where both $a_{i}$ and $f_{i}$ in the left stand for $k+1$ copies, for $i$ running from 0 to $k$. One easily checks that both $f_{k} a_{0^{-}}$ paths can be replaced by an $a_{k} f_{k}$-path of lower weight, while for all other rules $\ell \rightarrow r$ all $\ell$-paths can be replaced

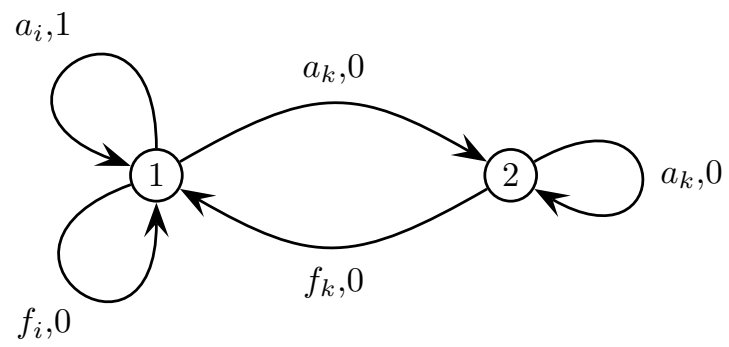
by an $r$-path of the same weight. So the rule $f_{k} a_{0} \rightarrow a_{k} f_{k}$ may be removed. Next remove $a_{k} f_{0} \rightarrow f_{k-1} a_{0}$ by counting $a_{k}$. The remaining system now is $R_{k-1}$, on which the argument is repeated until all rules have been removed.

\section{End symbols}

In this section we show that for SRSs $R$ of a particular shape termination of $\rightarrow_{R}$ and termination of $\rightarrow_{R}$ coincide. The special shape is characterized by an 
end symbol: a symbol $\mathrm{E}$ that only occurs as the last element of left hand sides an right hand sides of rules. We show that any SRS can be transformed to an SRS of this special shape, preserving termination and derivational complexity. As a consequence, termination of $a_{R}$ is undecidable, and for every computable function $F$ an SRS $R$ exists such that $\mathrm{dc}_{R}(n)=\Omega(F(n))$.

Lemma 11 Let $R$ be an $S R S$ over $\Sigma$ and let $\mathrm{E} \in \Sigma$. Let $R^{\prime} \subseteq R$ consist of the rules of $R$ in which $\mathrm{E}$ does not occur. Assume

1. every rule of $R \backslash R^{\prime}$ is of the shape $u \mathrm{E} \rightarrow v \mathrm{E}$ for $u, v \in(\Sigma \backslash\{\mathrm{E}\})^{*}$,

2. $\rightarrow R^{\prime}$ is terminating, and

3. $\rightarrow_{R}$ is terminating.

Then $\hookrightarrow_{R}$ is terminating.

Proof. . Assume $\hookrightarrow_{R}$ admits an infinite reduction $\left[u_{1}\right]_{\circ} \rightarrow_{R}\left[u_{2}\right] \hookrightarrow_{R}\left[u_{3}\right] \hookrightarrow_{R} \cdots$. Then due to assumption 1 it contains steps with respect to $R \backslash R^{\prime}$, so the symbol $\mathrm{E}$ occurs in $u_{1}$, so there is a string of the shape $v_{1} \mathrm{E} \in\left[u_{1}\right]$. From $\left[u_{1}\right] \circ a_{R}\left[u_{2}\right]$ we conclude that $v_{1} \mathrm{E}=u^{\prime} u^{\prime \prime}$ where $u^{\prime \prime} u^{\prime}=\ell x$ for some rule $\ell \rightarrow r$ in $R$ and some $x \in \Sigma^{*}$ and $r x \in\left[u_{2}\right]$. If $u^{\prime \prime}=\epsilon$ we may also choose $u^{\prime}=\epsilon$ and $u^{\prime \prime}=\ell x$, so in all cases we may assume that $u^{\prime \prime}$ is non-empty and ends in $\mathrm{E}$.

As $u^{\prime \prime} u^{\prime}=\ell x$, and $\ell$ contains no $\mathrm{E}$ other than in its last position by assumption 1, from $u^{\prime \prime}$ ending in $\mathrm{E}$ we conclude that $u^{\prime \prime}=\ell y$ for some $y$, and $x=y u^{\prime}$. If $y$ is non-empty it ends in $\mathrm{E}$, if $y=\epsilon$ then $\ell$ ends in $\mathrm{E}$, and then by assumption 1 also $r$ ends in $\mathrm{E}$. In all cases $r y$ ends in $\mathrm{E}$. Write $u^{\prime} r y=v_{2} \mathrm{E}$, then $r x=r y u^{\prime} \simeq u^{\prime} r y=v_{2} \mathrm{E}$, so $v_{2} \mathrm{E} \in\left[u_{2}\right]$ since $r x \in\left[u_{2}\right]$.

Summarizing, from $v_{1} \mathrm{E} \in\left[u_{1}\right]$ we constructed $v_{2} \mathrm{E} \in\left[u_{2}\right]$ such that

$$
v_{1} \mathrm{E}=u^{\prime} u^{\prime \prime}=u^{\prime} \ell y \rightarrow_{R} u^{\prime} r y=v_{2} \mathrm{E} \in\left[u_{2}\right] .
$$

Repeating this construction yields the infinite reduction $v_{1} \mathrm{E} \rightarrow_{R} v_{2} \mathrm{E} \rightarrow_{R} v_{3} \mathrm{E} \rightarrow_{R}$ $\cdots$, contradicting assumption 3 .

Next we define a transformation $\phi$ on SRSs such that an SRS $R$ is terminating if and only if $\hookrightarrow_{\phi(R)}$ is terminating, exploiting Lemma 11. Moreover, this transformation preserves reduction lengths. For a signature $\Sigma$ we define $\Sigma^{\prime}=\left\{f^{\prime} \mid f \in \Sigma\right\}$ in which for every $f \in \Sigma$ the symbol $f^{\prime}$ is fresh. Apart from these $f^{\prime}$ s we introduce three more fresh symbols L, R, E, of which E will act as the end symbol from Lemma 11. Now for an SRS $R$ over $\Sigma$ the SRS $\phi(R)$ over $\Sigma \cup \Sigma^{\prime} \cup\{\mathrm{L}, \mathrm{R}, \mathrm{E}\}$ is defined to consist of the rules
(a) $\mathrm{RE} \rightarrow \mathrm{LE}$
(b) $f \mathrm{~L} \rightarrow \mathrm{L} f^{\prime}$ for all $f \in \Sigma$
(c) $\mathrm{R} f^{\prime} \rightarrow f \mathrm{R}$ for all $f \in \Sigma$
(d) $\quad \ell \mathrm{L} \rightarrow r \mathrm{R}$ for all $\ell \rightarrow r \in R$

Theorem 12 In the above setting, for any SRS $R$ over $\Sigma$ the following three properties are equivalent: 
1. $\rightarrow_{R}$ is terminating,

2. $\rightarrow \phi(R)$ is terminating,

3. $\multimap \phi(R)$ is terminating.

Moreover, if a string $u \in \Sigma^{*}$ admits $a \rightarrow_{R}$ reduction of $n$ steps for some $n$, then $u \mathrm{RE}$ admits $a \rightarrow \phi(R)$ reduction of $n$ steps.

For proving this theorem we first need a lemma.

Lemma 13 Let $B C$ consist of the rules of type $(b)$ and $(c)$ above. Then $\hookrightarrow B C$ is terminating.

Proof. Similar to Example 8 we apply Theorem 4 using the following type graph, in which $f$ stands for all $f \in \Sigma$ and $f^{\prime}$ stands for all $f^{\prime}$ for which $f \in \Sigma$. Now by choosing $R=B C$ and $R^{\prime}=\emptyset$ all requirements of Theorem 4 hold, hence proving that $\rightarrow B C$ is terminating.

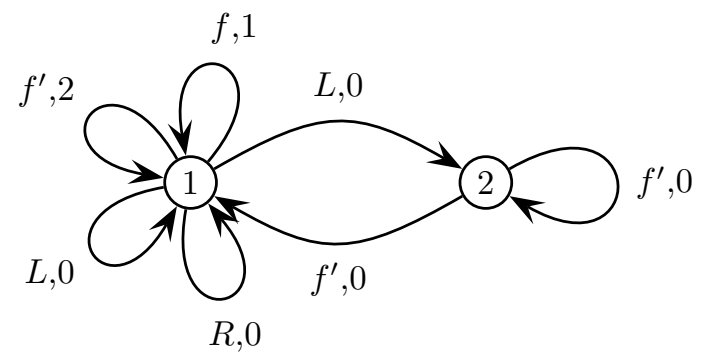

Now we arrive at the proof of Theorem 12 .

Proof. $1 \Rightarrow 2$ :

Assume $\rightarrow_{R}$ is terminating and $\rightarrow_{\phi(R)}$ admits an infinite reduction. If this infinite $\rightarrow \phi(R)$ reduction contains only finitely many $(d)$ steps, then there is an infinite $\rightarrow_{\phi(R)}$ reduction that only consists of $(a),(b),(c)$ steps. Then by counting $\mathrm{R}$ symbols there is also an infinite $\rightarrow_{\phi(R)}$ reduction only consisting of $(b),(c)$ steps, contradicting Lemmas 1, 13. So the infinite $\rightarrow_{\phi(R)}$ reduction contains infinitely many $(d)$ steps. In this reduction remove every $L$ symbol and every $\mathrm{R}$ symbol, and replace every symbol $f^{\prime}$ by $f$, for every $f \in \Sigma$. Then every $(a),(b),(c)$ step is replaced by an equality and every $(d)$ steps is replaced by an $R$ step, yielding an infinite $\rightarrow_{R}$ reduction, contradiction.

$2 \Rightarrow 1$ :

An infinite $\rightarrow_{R}$ reduction is transformed to an infinite $\rightarrow_{\phi(R)}$ by putting RE behind and the following observation, also proving the 'moreover' remark in the theorem:

if $u \rightarrow_{R} v$ then $u \mathrm{RE} \rightarrow_{\phi(R)}^{+} v \mathrm{RE}$.

This is shown as follows: write $u=x \ell y$ and $v=x r y$ for $\ell \rightarrow r \in R$, then

$$
u \mathrm{RE}=x \ell y \mathrm{RE} \rightarrow{ }_{(a)} x \ell y \mathrm{LE} \rightarrow_{(b)}^{*} x \ell \mathrm{L} y^{\prime} \mathrm{E} \rightarrow{ }_{(d)} x r \mathrm{R} y^{\prime} \mathrm{E} \rightarrow_{(c)}^{*} x r y \mathrm{RE}=v \mathrm{RE} .
$$

$3 \Rightarrow 2$ : Immediate from Lemma 1 .

$2 \Rightarrow 3$ :

We apply Lemma 11 on the SRS $\phi(R)$. First observe that $\phi(R)$ satisfies condition 1 by construction. So it remains to prove condition 2 : the rules of type $(b),(c),(d)$ are terminating. By counting $\mathrm{L}$ symbols it suffices to prove that the rules of type $(b),(c)$ are terminating, which follows from Lemma 13. 
Theorem 14 Termination of $a_{R}$ is an undecidable property, and for every computable function $F$ an $S R S R$ exists such that $\mathrm{dc}_{R}(n)=\Omega(F(n))$.

Proof. The main result from [5] states that every Turing machine $M$ can be transformed to an SRS $R_{M}$ such that $\rightarrow_{R_{M}}$ is terminating if and only if $M$ halts on every input, proving that termination of string rewriting is undecidable. Applying Theorem 12 we obtain that that every Turing machine $M$ can be transformed to an SRS $\phi\left(R_{M}\right)$ such that $\hookrightarrow{ }_{\phi\left(R_{M}\right)}$ is terminating if and only if $M$ halts on every input. So also termination of $\circ \rightarrow$ is undecidable.

For the second claim take a uniformly halting Turing machine $M$ such that for every $n$ there is a configuration of size $O(n)$ admitting $\Omega(F(n))$ transitions before halting; a Turing machine computing $F(n)$ satisfies this property. Now due to Theorem $12 \hookrightarrow_{\phi\left(R_{M}\right)}$ is terminating and satisfies dc d $_{\phi\left(R_{M}\right)}(n)=\Omega(F(n))$.

\section{Implementation}

We implemented all techniques presented in this paper in our tool TORPAcyc. More precisely, for a given SRS the tool tries to prove termination stepwise by tropical and arctic type graphs for increasing graph size running from 1 to 3 . As soon a rule is found yielding a strict decrease while all other rules yield a weeks decrease, this rule is removed and the process continues with the rest. For graph size 1 this corresponds to simple weight arguments, so this is by what the procedure always starts. Apart from this also the match-bound method is applied, in which the corresponding type graph is constructed by completion, typically yielding graphs of up to hundreds of nodes.

For match-bounds the implementation from the tool TORPA has been reused, as presented in [9]. For searching for type graphs the real work is done by the external SMT solver Yices: the requirements are expressed in an SMT formula similar to other implementations of arctic and tropical matrix interpretations, and whenever Yices finds a satisfying assignment, the corresponding type graph is constructed from this satisfying assignment and presented in matrix notation.

A zip file containing the source code, a Linux executable, a parameter file, the external tool Yices and several examples can be downloaded from

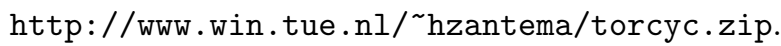

The parameter file param contains several parameters which may be edited. For instance, if you want to find a type graph proof with weights as small as possible, you may stepwise decrease the maximal value for type graph weights.

\section{Conclusions}

Syntactically cycle rewriting is the same as string rewriting, but the semantics is different: as in cycle rewriting the start of the string is connected to the end, more rewrite steps are possible, and the notion of termination of cycle rewriting 
is strictly stronger than termination of string rewriting. Techniques for proving termination of string rewriting based on monotone algebras and dependency pairs strongly exploit the structure of strings having a start and an end, and do not serve for modifications proving termination of cycle rewriting. In this paper we showed that tropical matrix interpretations, arctic matrix interpretations and match-bounds apply for proving termination of cycle rewriting. These techniques are the same that are used for proving linear derivational complexity for term and string rewriting in [8]. Although the requirements for these techniques are the same as for proving termination of string rewriting, the proofs that they also serve for proving termination of cycle rewriting is quite different. The standard approach for matrix methods for string rewriting is based on monotone algebras, while our approach for cycle rewriting makes use of morphisms of strings into type graphs. Our result for match-bounds follows from observing that match-bounds are a special case of tropical matrix interpretations. Apart from only proving termination we also investigated derivational complexity of our techniques, and developed transformations by which we could show that termination of cycle rewriting is undecidable and every computable derivational complexity can be reached.

\section{References}

1. H.J.S. Bruggink, B. König, and H. Zantema. Termination analysis for graph transformation systems. submitted, available at http://www.ti.inf.uni-due.de/fileadmin/public/bruggink/gts-termination.pdf, 2014 .

2. A. Corradini, U. Montanari, and F. Rossi. Graph processes. Fundamenta Informaticae, 26(3/4):241-265, 1996.

3. J. Endrullis, J. Waldmann, and H. Zantema. Matrix interpretations for proving termination of term rewriting. Journal of Automated Reasoning, 40:195-220, 2008.

4. A. Geser, D. Hofbauer, and J. Waldmann. Match-bounded string rewriting systems. Applicable Algebra in Engineering, Communication and Computing, 15(3-4):149171, 2004.

5. G. Huet and D. S. Lankford. On the uniform halting problem for term rewriting systems. Rapport Laboria 283, INRIA, 1978.

6. A. Koprowski and J. Waldmann. Arctic termination ... below zero. In A. Voronkov, editor, Proceedings of the 19th Conference on Rewriting Techniques and Applications (RTA), volume 5117 of Lecture Notes in Computer Science, pages 202-216. Springer, 2008.

7. I. Simon. Recognizable sets with multiplicities in the tropical semiring. In Mathematical Foundations of Computer Science, volume 324 of Lecture Notes in Computer Science, pages 107-120. Springer, 1988.

8. H. Zankl and M. Korp. Modular complexity analysis via relative complexity. In Proceedings of the 21st Conference on Rewriting Techniques and Applications (RTA), pages 385-400. Lipics, 2010.

9. H. Zantema. Termination of string rewriting proved automatically. Journal of Automated Reasoning, 34:105-139, 2004. 